\title{
Pulling and Pushing Stem Cells to Control
}

\section{differentiation.}

Nureddin Ashammakhi ${ }^{1, *}, \mathrm{MD}, \mathrm{FRCSEd}, \mathrm{PhD}$

Outi Kaarela ${ }^{1}, \mathrm{MD}, \mathrm{PhD}$

Patrizia Ferretti ${ }^{2}, \mathrm{PhD}$

${ }^{1}$ Division of Plastic Surgery, Department of Surgery, Oulu University, Oulu, Finland.

${ }^{2}$ Professor of Regenerative Biology, UCL Institute of Child Health (University College London)

UCL, London, United Kingdom

(*) Corresponding author

Nureddin Ashammakhi

Department of Surgery, Oulu University Hospital, P.O. Box 22, FI-90220 Oulu, Finland

Tel. +358 8 3152011, E-mail: nureddin.ashammakhi@oulu.fi 


\begin{abstract}
With advances made in various directions of regenerative medicine including the use of stimuli-responsive materials, 4D biofabrication, inducible pluripotent stem cells (iPSCs), control of stem cell fate using chemical and physical factors, minimal access delivery, and information-communication technology, much can be done to achieve preciselycontrolled and customised regenerative therapy. In this short perspective, recent advances are discussed, synthesised with focus on discussing recent report on the use of mechanical stretching of nanoparticle-laden stem cells by using external magnetic field towards defined cardiac line differentiation. Although, more tools are becoming available to use, our capabilities are stronger, and range of applications is expanding, there is much work that needs to be done before clinical applications can successfully be achieved.
\end{abstract}

Keywords. Magnetic, Nanoparticles, Regenerative medicine, Stem cell differentiation. 


\section{Introduction}

Tissue defects and organ failure need repair reconstruction of their structure and reconstitution of function. It is often difficult to obtain enough tissue for transplantation. The use of stem cells has revolutionised our approaches to engineer tissues, and stemcell based therapy promises to develop personalised medicine in future $(1,2)$. Pluripotent stem cells (PSCs) whether embryonic (ESCs) or induced pluripotent (iPSCs) stem cells are of great importance in developing tailored regenerative personalised medicine. Attempts to control the fate of stem cells continue to be a challenge yet appealing to investigate by using various chemical, physical or combinational cues.

\section{Control of stem cell fate by mechanical cues}

Magnetic particles were previously used for moving cells to their target destination. The use of mechanical cues to control stem cell differentiation became recently more attractive. Guiding stem cell differentiation by using mechanical stimuli solely, was recently reported. External magnetic field was employed for pulling iron oxide nanoparticle-laden stem cells to form embryoid body. This led to pushing stem cells towards cardiac line differentiation by exerting mechanical stretching using opposing magnetic fields (Figure 1). Du et al. (3) showed that magnetic nanoparticles internalized by ESCs can efficiently be used to stretch and move cells into aggregates and form embryoid bodies (EBs). The process can be achieved by using remotely-controlled opposing magnetic attractors.

\section{Du's study}

Du's study develops an alternative new method for the formation of embryoid bodies (EBs) which is better than the conventionally most commonly used one, i.e. hanging 
drop method which is time consuming involving multiple manipulations. Other classical methods include the use of hydrogel biomaterial for encapsulation, or spontaneous formation as suspension in culture. Compared to hanging drop method, the use of magnetic field enables tight control over the size of EBs.

EBs were formed from 10,000 cells. After $1 \mathrm{~h}$, stretching opposite field was applied. Stretched status was maintained for the next 3 days. In addition, cyclic stimulation was achieved by applying stretch for $2 \mathrm{~h}$ daily over the 3 days. Single-cell movement was monitored by using cell membrane marker. Magnets were then removed, and spheroids were let to mature until day 5 after which analysis was performed. EBs maintained their engineered shape. The three processing conditions (magnet, stretching and cyclic stimulation) were compared for their effect on differentiation gene expression (characteristic of different embryonic layers).

Mechanical stimulation alone led to cardiac lineage differentiation. There was a significant increase in the expression of 3 genes (Sox17, Gata4 and Gata6) which are indicators of the following stage in the pathway towards cardiac differentiation. There was also an increase in the expression of cardiac pathway gene (Nkx2.5) which was enhanced under cyclic conditions. Genes associated with ectodermal and endodermal differentiation were either down-regulated or lowly upregulated. There was also overexpression of genes related to final cardiac lineage commitment at day 10 , such as for Tnnt2 and Myh-6. Which were higher with cyclic stimulation. Cyclic stimulation was shown earlier to enhance cardiac differentiation and cardiomyocyte maturation $(4,5)$. Similarly, in Du's observation, cyclic stretching was associated with more expression of cardiac markers such as (troponin T (Tnnt2), involved in cardiomyocyte contraction, 
and $\alpha$ myosin heavy chain (Myh6), involved in contraction and considered as a maturation marker) as compared to magnet or stretch alone methods, mimicking cardiac contraction.

\section{Comment}

Mechanical stimulation was exerted on single-cell level through magnetic means via internalised nanoparticles. Subjecting each cell to magnetic force pulls cells with their neighbours and cohesion builds up subsequently through cell-cell junctions which is strong enough to keep EBs standalone. Potentially, this method can be used with other types of cells which can assemble and develop cohesion through cell-cell junctions. Such a smart strategy can thus help building scaffold-free 3D EBs in which ESCs differentiation can be mechanically influenced without needing to introduce chemical factors. Further studies and applications using other types of cells in regenerative therapy are encouraged to make use of this achievement.

Regarding concerns with the elimination of magnetic nanoparticles, about half of the nanoparticles have disappeared by seven days. Previous studies showed that they undergo removal by possibly lysosomal degradation $(6,7)$.

\section{Electromagnetic biomaterials}

In earlier works, magnetic iron oxide nanoparticles were used to bioassemble neural stem cells into 3D structures as an alternative method of using artificial matrix. With the help of external magnet, it was possible to levitate cells into aggregates at the surface of the medium (8) (Figure 2). 
Magnetic nanoparticle-laden bone-marrow derived mesenchymal stem cells (BMSCs) were used to generate cell spheroids in reproducible and size-controlled fashion by using magnetic pins that were induced to generate concentrated magnetic field and attract particle-laden cells towards them (9) (Figure 3).

The use of elctroconductive materials such as gold can also be used to help cardiac tissue engineering, e.g. gold nanorods incorporated in a bioprinting hydrogel were used for improving synchronisation of contraction of cardiac myocytes (10). This can possibly be added in next stage to augment the function of engineered cardiac tissue. One has to look into combinational approaches for the best outcome towards efficient therapy that can be applied with success in treatment of conditions such as MI.

\section{Other tools}

With stimuli-responsive materials (11-13), more possibilities will open not only for drug delivery but also for advanced regenerative medicine and for $4 \mathrm{D}$ constructs in which form can change after implantation in response to internal or external stimuli. Examples include the stimuli responsive shape memory-based mesh for delivery of cardiac patch experimented for the treatment of myocardial infarction (14). Even gels can be used as shape memory materials (15). Similarly, cell contraction may be used in controlling material folding in cellular origami(16).

Application would include other types of cells which are sensitive to mechanical stimulation such as osteogenic, chondral, myogenic, tendon, etc. It can also be provided in tailored and graduated fashion in different cell types in engineering different types of tissues, engineering, etc. Recent advances in microfluidic devices and the use of organ- 
on-a-chip can benefit from this achievement and develop the technology further for studies of physiology, pathological models and drug testing and development.

Aided by microfluidic channels, it is possible to trap (17), capture-and-release (18), divide (19), encapsulate (20-24) and use for bioprinting (21) laying basis for further possibilities of genetic treatment and cell manipulation at the micro- and submicron levels. The use of single cell encapsulation will enable more possibilities of control of stem cells by inclusion of chemical factors and gradient building (25) in resulting cellbiomaterial construct. In a recent report, drug delivery to skin was externally controlled by linking to mobile phone app. (26). These tools combined will make our capabilities in future not only diversified, but exponentially advanced and application areas expanded.

\section{Conclusions}

Development of cell spheroids is important tool to deliver regenerative therapies. Control of stem cell fate represents and advancing frontier and it was possible to achieve by using mechanical cues solely. Microfluidic techniques allow combining various factors and single cell encapsulation. Use of memory shape materials will help cell delivery. Several mechanisms are thus possible today to exert mechanical and chemical impact and may be combined to influence behaviour, function and fate of stem cells towards new generation of $4 \mathrm{D}$ regenerative strategies.

\section{Disclosure}

No conflict of interest statement. 
References

1. Wimpenny I, Hampson K, Yang Y, Ashammakhi N, Forsyth NR. One-step recovery of marrow stromal cells on nanofibers. Tissue Eng Part C Methods. 2010 June 01;16(3):503-9.

2. Bajada S, Mazakova I, Richardson JB, Ashammakhi N. Updates on stem cells and their applications in regenerative medicine. J Tissue Eng Regen Med. 2008 June 01;2(4):169-83.

3. Vicard Du, Nathalie Luciani, Sophie Richard, Gaëtan Mary, Cyprien Gay, François Mazuel, Myriam Reffay, Philippe Menasché, Onnik Agbulut \& Claire Wilhelm. A 3D magnetic tissue stretcher for remote mechanical control of embryonic stem cell differentiation. Nat Com. 2017.

4. Gwak S, Bhang SH, Kim I, Kim S, Kim B, Cho S, et al. The effect of cyclic strain on embryonic stem cell-derived cardiomyocytes. Biomaterials. 2008;29(7):844-56.

5. Zhang T, Wan LQ, Xiong Z, Marsano A, Maidhof R, Park M, et al. Channelled scaffolds for engineering myocardium with mechanical stimulation. Journal of Tissue Engineering and Regenerative Medicine. 2012 Oct;6(9):748-56.

6. Mazuel F, Espinosa A, Luciani N, Reffay M, Le Borgne R, Motte L, et al. Massive Intracellular Biodegradation of Iron Oxide Nanoparticles Evidenced Magnetically at Single-Endosome and Tissue Levels. ACS nano. 2016 Aug 23,;10(8):7627-38.

7. Mazuel F, Espinosa A, Radtke G, Bugnet M, Neveu S, Lalatonne Y, et al. Magneto-Thermal Metrics Can Mirror the Long-Term Intracellular Fate of Magneto-Plasmonic Nanohybrids and Reveal the Remarkable Shielding Effect of Gold. Advanced Functional Materials. 2017 Mar 3,;27(9):n/a.

8. Souza GR, Molina JR, Raphael RM, Ozawa MG, Stark DJ, Levin CS, et al. Three-dimensional tissue culture based on magnetic cell levitation. Nat Nanotechnol. 2010 April 01;5(4):291-6.

9. Kim JA, Choi J, Kim M, Rhee WJ, Son B, Jung H, et al. High-throughput generation of spheroids using magnetic nanoparticles for three-dimensional cell culture. Biomaterials. 2013 Nov;34(34):8555-63.

10. Zhu K, Shin SR, van Kempen T, Li Y, Ponraj V, Nasajpour A, et al. Gold Nanocomposite Bioink for Printing 3D Cardiac Constructs. Advanced Functional Materials. 2017 Mar 24,;27(12):n/a.

11. Ashammakhi N, Kaarela O. Stimuli-Responsive Biomaterials: Next Wave. J Craniofac Surg. 2017 Oct;28(7):1647-8.

12. Lu Y, Aimetti AA, Langer R, Gu Z. Bioresponsive materials. Nature Reviews Materials. 2016 Oct $25, ; 2(1): 16075$.

13. Culver HR, Clegg JR, Peppas NA. Analyte-Responsive Hydrogels: Intelligent Materials for Biosensing and Drug Delivery. Acc. Chem. Res. 2017;50:170-178.

14. Montgomery $\mathrm{M}$, et al. Flexible shape-memory scaffold for minimally invasive delivery of functional tissues. Nature Materials. 2017.

15. Kirillova A, Maxson R, Stoychev G, Gomillion CT, lonov L. 4D Biofabrication Using ShapeMorphing Hydrogels. Adv. Mater. 2017. 
16. Kuribayashi-Shigetomi K, Onoe H, Takeuchi S. Cell origami: self-folding of three-dimensional cellladen microstructures driven by cell traction force. PloS one. 2012;7(12):e51085.

17. Thomas RS, Mitchell PD, Oreffo RO, Morgan H. Trapping single human osteoblast-like cells from a heterogeneous population using a dielectrophoretic microfluidic device. Biomicrofluidics. 2010 June 29;4(2):10.1063/1.3406951.

18. Yoon HJ, Shanker A, Wang Y, Kozminsky M, Jin Q, Palanisamy N, et al. Tunable Thermal-Sensitive Polymer-Graphene Oxide Composite for Efficient Capture and Release of Viable Circulating Tumor Cells. Adv Mater. 2016 June 01;28(24):4891-7.

19. Blauch LR, Gai Y, Khor JW, Sood P, Marshall WF, Tang SKY. Microfluidic guillotine for single-cell wound repair studies. Proc Natl Acad Sci U S A. 2017 July 11;114(28):7283-8.

20. Lienemann PS, Rossow T, Mao AS, Vallmajo-Martin Q, Ehrbar M, Mooney DJ. Single cell-laden protease-sensitive microniches for long-term culture in 3D. Lab Chip. 2017 February 14;17(4):727-37.

21. Kamperman T, Henke S, van den Berg A, Shin SR, Tamayol A, Khademhosseini A, et al. Single Cell Microgel Based Modular Bioinks for Uncoupled Cellular Micro- and Macroenvironments. Adv Healthc Mater. 2017 February 01;6(3):10.1002/adhm.201600913. Epub 2016 Dec 14.

22. Mao AS, Shin JW, Utech S, Wang H, Uzun O, Li W, et al. Deterministic encapsulation of single cells in thin tunable microgels for niche modelling and therapeutic delivery. Nat Mater. 2017 February 01;16(2):236-43.

23. Eric Brouzes, Martina Medkova, Neal Savenelli, Dave Marran, Mariusz Twardowski, J. Brian Hutchison, et al. Droplet Microfluidic Technology for Single-Cell High-Throughput Screening. Proceedings of the National Academy of Sciences of the United States of America. 2009 Aug $25, ; 106(34): 14195-200$.

24. Ma Y, Neubauer MP, Thiele J, Fery A, Huck WTS. Artificial microniches for probing mesenchymal stem cell fate in 3d. Biomaterials Science. 2014;2(11):1661-71.

25. Batzaya Byambaa, Nasim Annabi, Kan Yue, Grissel Trujillo-de Santiago, Mario Moisés Alvarez, Weitao Jia, et al. Bioprinted Osteogenic and Vasculogenic Patterns for Engineering 3D Bone Tissue. 2017.

26. Mostafalu P. A Textile Dressing for Temporal and Dosage Controlled Drug Delivery. Adv Funct Mater. 2017.

27. Nakanishi M, Kurisaki A, Hayashi Y, Warashina M, Ishiura S, Kusuda-Furue M, et al. Directed induction of anterior and posterior primitive streak by Wnt from embryonic stem cells cultured in a chemically defined serum-free medium. FASEB journal : official publication of the Federation of American Societies for Experimental Biology. 2009 Jan;23(1):114-22.

28. Willems E, Bushway P, Mercola M. Natural and Synthetic Regulators of Embryonic Stem Cell Cardiogenesis. Pediatr Cardiol. 2009 Jul;30(5):635-42.

29. Omidinia-Anarkoli A, Boesveld S, Tuvshindorj U, Rose JC, Haraszti T, De Laporte L. An Injectable Hybrid Hydrogel with Oriented Short Fibers Induces Unidirectional Growth of Functional Nerve Cells. Small. 2017 Aug 7. 
30. Li J, Zhang W, Chung TF, Slipchenko MN, Chen YP, Cheng JX, et al. Highly sensitive transient absorption imaging of graphene and graphene oxide in living cells and circulating blood. Sci Rep. 2015 July 23;5:12394.

\section{Figure legends}

Figure 1. Use of magnetic nanoparticles for control of cell organisation and differentiation.

(A) Schematic illustration of magnetic stretching: i. Nanoparticles were incorporated into embryonic stem cells (ESCs). ii. Embryonic body (EB) formation with the help of a magnetic microtip, and iii. EBs magnetic stretching of EB.

(B) i. Monitoring of EBs magnetism over seven days following EB formation. Scale bar is $200 \mu \mathrm{m}$. ii. Illustration of use of magnet to move EBs, in which velocity of EB migration towards magnet is determined by balancing the viscous drag force $\left(\mathrm{F}_{\mathrm{DRAG}}\right)$ and the magnetic force ( $\mathrm{F}_{\mathrm{MAG}}$ ). Original figure was adapted from Nakanishi et al. (27) and Willems et al. (28). Reproduced with kind permission from Du et al. (3).

Figure 2. Use of magnetic nanoparticles for cell aggregation and levitation.

(A) Three-dimensional (3D) cell culture with magnetic-based levitation illustration (top row) and micrographs (bottom row) showing: i. Cell-containing hydrogel is incubated; ii. Removal of non-interacting hydrogel fragments by washing; Nanoparticles enter cells or are membrane-bound; iii. Cells rise to the surface of the medium by the effect of externally-applied magnet; iv. Multicellular aggregates are formed, $12 \mathrm{~h}$ following levitation. Scale bar (in the bottom row) is $30 \mu \mathrm{m}$. (B) Control of the shape of levitated cells, with magnets having an outer radius of $12 \mathrm{~mm}$ (i-iii) or $6 \mathrm{~mm}$ (iv-vi). Cells at the 
start of levitation (i, iv), 30h (ii, v) and at 30h but following magnet removal (iii, vi). Reproduced with kind permission from Souza et al. (8).

Figure 3. Use of magnetic and gold nanoparticles/rods for cell assembly, guidance and function.

(A) Creation of magnetic nanoparticle-laden bone-marrow derived mesenchymal stem cell (BMSCs) spheroids, by using magnetic pins. Reproduced with kind permission from Kim et al. (9). (B) Use of magnetic short fibers in a hydrogel that can be aligned using external magnetic field, after which gel is crosslinked in situ and fibers are maintained in their oriented position, in order to induce unidirectional growth of nerve cells.

Reproduced with permission from Omidinia-Anarkoli et al. $(29,30)$.

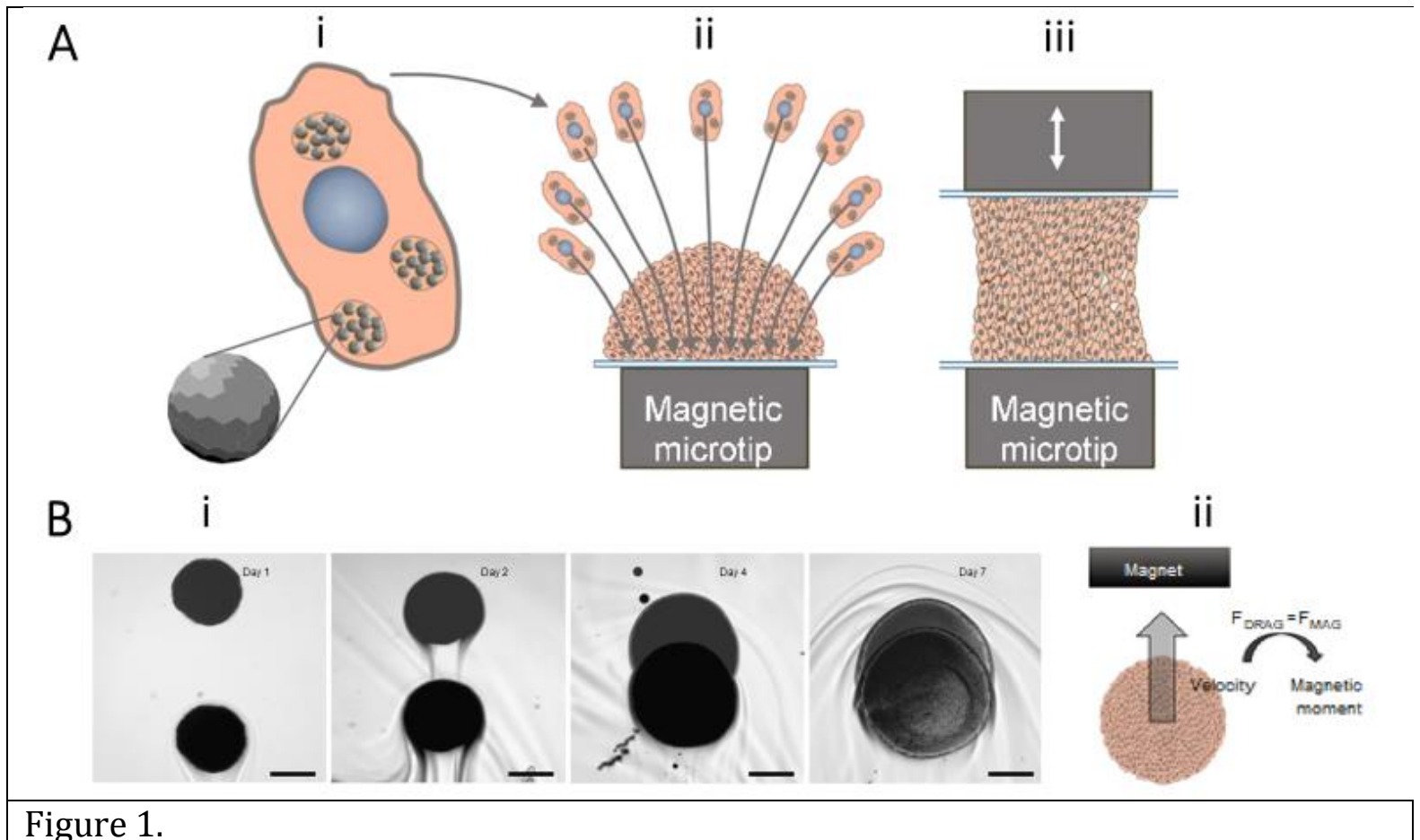

Figure 1. 
A

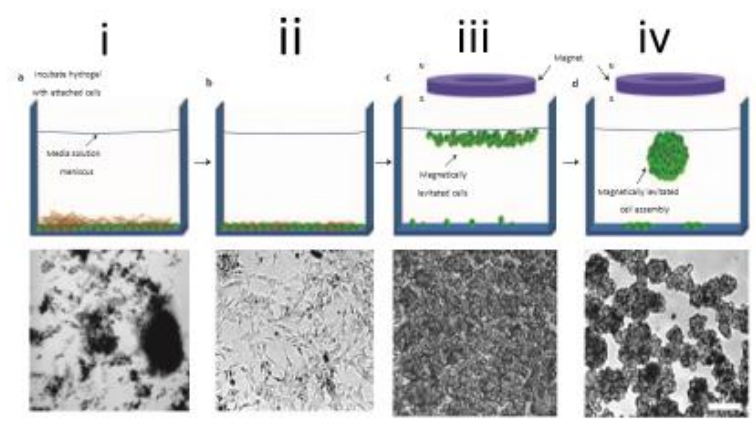

B

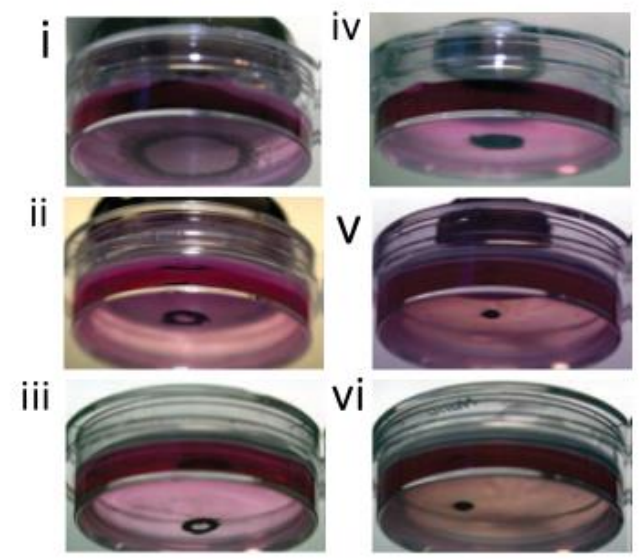

Figure 2.

A

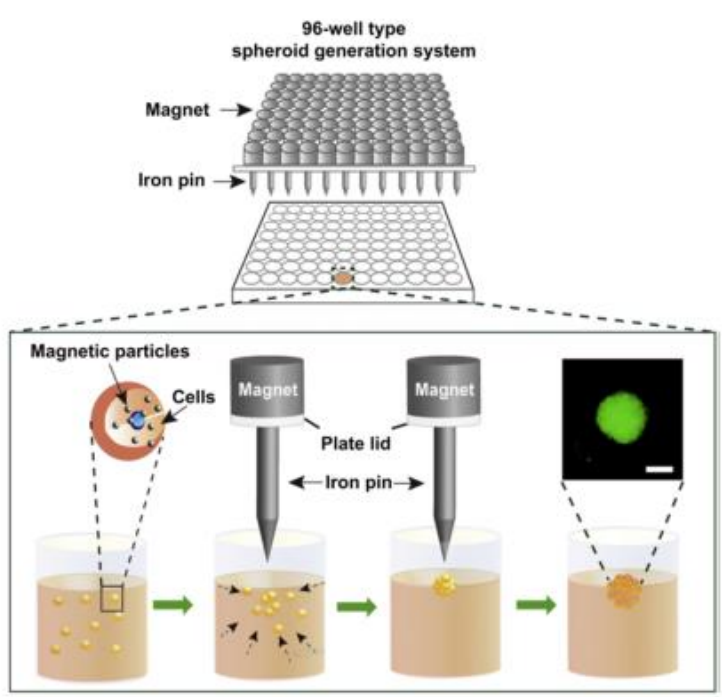

B

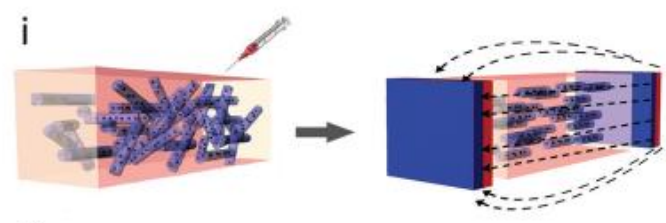

ii

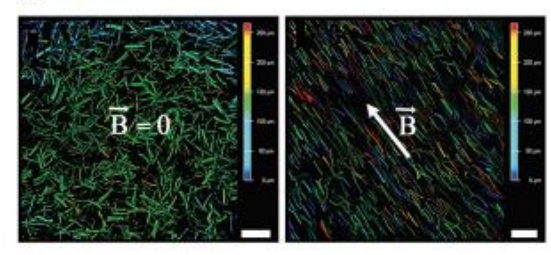

iii

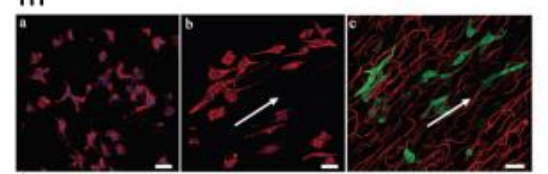

Figure 3. 